22 for anisotropy in the distribution of cracks, an eigenanalysis is also performed. The

23 results show that micro-CT can be a useful tool for both visualizing and quantifying

24 damage in polycrystalline ice and that a 3-D analog of the traditional second-rank crack

25 density tensor can be readily calculated via commercially available software. Kevin Hammonds ${ }^{1,2} \&$ Ian Baker $^{1}$

${ }^{1}$ Thayer School of Engineering at Dartmouth College, Hanover, NH, USA ${ }^{2}$ Montana State University, Department of Civil Engineering, Bozeman, MT, USA

\section{ABSTRACT}

The use of X-ray computed micro-tomography (micro-CT) is presented here as a useful tool for the analysis and quantification of damage in polycrystalline ice. Although known to be useful for characterizing damage in many other materials, the use of microCT has not yet been adapted to the non-trivial case of also characterizing damage in polycrystalline ice. Samples of polycrystalline ice were tested in uniaxial compression at six different strain rates, spanning four orders of magnitude, from $1 \times 10^{-6} \mathrm{~s}^{-1}$ to $1 \times 10^{-3} \mathrm{~s}^{-1}$, and two different testing temperatures of $-10^{\circ} \mathrm{C}$ and $-20^{\circ} \mathrm{C}$. The extent of cracking from each test is characterized via micro-CT imaging and is quantified via a newly proposed variant of the crack density tensor, which accounts for any anisotropy in the mean crack orientation and is shown to be equivalent to the materials anisotropy tensor. To account

\title{
Quantifying Damage in Polycrystalline Ice via X-Ray Computed Micro-tomography
}


52 CORRESPONDING AUTHOR

53

54 Kevin Hammonds

55

56 Montana State University

57

58 Department of Civil Engineering

59

60205 Cobleigh Hall

61

62 P.O. Box 173900

63

64 Bozeman, MT 59717

65

$66 \quad 406-994-2167$ (office)

67

68 Kevin.Hammonds@Montana.edu 


\section{INTRODUCTION}

Over the last two decades, the use of X-ray computed micro-tomography (micro-

72 CT) has become a widely used laboratory technique for many applications in materials

73 science and engineering [1,2]. Underlying much of this broad appeal is the ability of

74 micro-CT to nondestructively characterize material microstructures at a micron resolution

75 in three dimensions. To date, micro-CT has been successfully employed to characterize

76 an extensive variety of materials and processes, such as bone porosity [3], brine channels

77 and air inclusions in sea ice [4], the fracture and damage of concrete [5], the

78 microstructural evolution of snow under a temperature gradient [6], fatigue crack

79 propagation in cast-iron [7], etc. Although not nearly an exhaustive list, one area of study

80 where the advantages of micro-CT have not yet been fully realized, is the study and 3-D

81 characterization of damage in strained polycrystalline ice. Developing such an

82 understanding is thought to be of critical importance to better predicting the fracture

83 mechanics of both land-based and sea-based ice flows and is thus the primary focus of

84 this work.

85 Like many other materials, ice can sustain damage in the form of non-propagating

86 cracks that result from localized strains [8]. The presence of these cracks, in turn, can

87 have dramatic affects on the material properties of the ice, even though the larger body

88 remains fully intact. Understanding the role of deformation and damage in

89 polycrystalline ice, including columnar ice, has been the topic of many laboratory, field,

90 and theoretical studies spanning the past five decades $[9,10,11,12,13,14,15,16]$. This

91 interest is derived from the broad application of such knowledge, including calving rates

92 of glaciers and ice sheets [17,18], predicting sea ice behavior in the Arctic and Antarctic 
93 oceans $[19,20,21,22]$, structural engineering of off-shore structures in the polar regions

$94[23,24,25]$, and interpretation of the surface characteristics of icy terrestrial bodies $95[26,27,28]$.

96 In order to quantify and validate the predictions of traditional fracture mechanics,

97 as it would apply to ice $\mathrm{I}_{\mathrm{h}}$, there is often an imaging component of the analysis required

98 for either the investigation of field-collected samples or laboratory tested specimens.

99 This imaging component requires that the actual cracks be imaged such that they can be

100 manually counted, measured, and characterized. This is an important component of the

101 analysis, as the size, type, and number of cracks in a sense act as benchmarks detailing

102 the mechanical history of the ice. For example, the observation of "comb-cracks" versus

103 "wing-cracks" along a fault may be indicative of the destabilization mechanism of the

104 body [15], whereas evidence of intergranular versus transgranular fracture may be more

105 telling about the response of the ice to either elastic or plastic deformation [29], 106 respectively.

107 Similar to many polycrystalline metals and alloys, the study of polycrystalline ice

108 via micro-CT has previously been thought of as a poor choice of materials for micro-CT

109 analysis as no other microstructural information can be elucidated and x-ray attenuation

110 in ice can be quite high [4]. Given sufficient voiding, cracking, or pore space is present

111 within the material, the use of polychromatic x-ray sources has been shown to be useful,

112 mostly limited only by camera resolution. This has been evidenced by other recent

113 micro-CT studies pertaining to sea ice inclusions [4], the bonding of ice spheres [30], and

114 the metamorphism of ice-snow interfaces [31]. 


\subsection{Crack Density Tensor}

118 A very recent and detailed review of the various ways to measure and quantify

119 damage in polycrystalline ice is given in Snyder et al. (2015) [8] and is not repeated here.

120 To summarize, however, most approaches involve first the concession of a 3-D

121 perspective to a 2-D perspective, such that all cracks within a 2-D plane can be

122 differentiated and then manually measured and counted. Making such measurements can

123 be quite tedious as both the crack length and the normal orientation of the crack from a

124 fixed point of reference is required. With this information, a dimensionless 2-D crack

125 density tensor $\alpha_{2-D}$ can be derived

$$
\alpha_{2-D}=\frac{1}{A} \sum_{i}\left(c^{2} \vec{n} \vec{n}\right)_{i}
$$

129 where $A$ is the cross-sectional area of the sample, $c$ is the crack half-length, and $\vec{n}$ is the

130 unit vector normal to the $i$ th crack of length $2 c[32,8]$. The vector product $\vec{n} \vec{n}$ denotes the

131 dyadic, which when summed over all cracks and all orientations, gives a measure of the

132 anisotropy of the measured crack orientation in a tensorial format as a second-rank tensor

133 [8]. If extending to the 3-D case, Eq. (1) simply becomes

$$
\alpha_{3-D}=\frac{1}{V} \sum_{i}\left(a^{3} \vec{n} \vec{n}\right)_{i}
$$

137 where $V$ is the sample volume and $a$ is the radius of the $i$ th circular-shaped crack $[33,8]$.

138 In either format, the benefit of the tensorial representation is that it lends a continuum 
139 approach to understanding the damage mechanics of the material [34]. In the case of an

140 isotropic or random distribution of crack orientations, Eq. (1) and Eq. (2) simplify to the

141 scalar (first invariant of the tensor), which is the trace of alpha $\left(\operatorname{tr}(\alpha)=\rho_{c}\right)$.

142

$143 \quad 2.2$ Stereological Analysis

144 Of the many variables that can be calculated with the Bruker CTan software, the

145 3-D stereological analysis is focused on here as perhaps the most beneficial in handling

146 anisotropy and crack density distributions. Originally developed for the analysis of pore

147 space in bone, the stereological analysis makes use of a statistical technique in which a

148 mean intercept length is calculated in 3-D from within a spherical volume encompassing

149 the user-defined volume of interest (VOI). Within this encompassing sphere, lines can be

150 drawn in up to 1024 different orientations with a spacing that is user-defined, but can be

151 as small as one pixel or "voxel" (a 3-D pixel) apart. Once drawn, the orientation of each

152 line is recorded and the length of each line is divided by the number of times it intercepts

153 an object (voxels equal to 1 on a binary scale), this quotient defines the mean intercept

154 length $l$. It should be noted, however, that whether or not the line drawn traverses a lone

1551 pixel or ten 1 pixels, as long as these pixels are connected, this would only count as one

156 intercept. Thus, the mean intercept length could also be thought of as a metric for the

157 length between cracks and not necessarily the crack length itself.

158 For the mean intercept length analysis presented in this study, the number of 159 randomly selected orientations was set to 512 with a $90 \mu \mathrm{m}$ (5 pixel) spacing. For 160 perspective, this allowed for 53,625 lines to be drawn per orientation within a VOI of

$1614213 \mathrm{~mm}^{3}$. Once complete, the number and length of all intercepts over all orientations 
162 are related back to the principal axes of the encompassing sphere, such that three

163 principal mean intercept lengths, $l_{1}, l_{2}$, and $l_{3}$ and vector orientations, $\vec{e}_{1}, \vec{e}_{2}$, and $\vec{e}_{3}$ can

164 be determined. These orientations are solved for via the statistical fitting of an ellipsoid

165 encompassing all mean intercept length values when each intercept is plotted as a radius

166 with each line anchored at the origin. Originally given in Harrigan and Mann (1984)

167 [36], this process creates the surface of an ellipsoid of the general form

168

$$
A x_{1}^{2}+B x_{2}^{2}+C x_{3}^{2}+2 D x_{1} x_{2}+2 E x_{1} x_{3}+2 F x_{2} x_{3}=1
$$

where $A, B, C, D, E$, and $F$ are dimensionless coefficients and $x_{1}, x_{2}$, and $x_{3}$ are

172 the principal axes of the original Cartesian coordinate system of the VOI. $\vec{e}_{1}, \vec{e}_{2}$, and $\vec{e}_{3}$

173 can then be solved for via an eigenanalysis, determining the rotation of the orthogonal

174 principal axes of the ellipsoid from the original axes of the VOI, such that $\vec{e}_{1}, \vec{e}_{2}$, and $\vec{e}_{3}$

175 are the eigenvectors representing the orientations of $l_{1}, l_{2}$, and $l_{3}$ relative to the original

176 Cartesian coordinate system of the VOI. Additionally, the eigenvalues $E_{1}, E_{2}$, and $E_{3}$,

177 from the eigen analysis can be used as a measure of the degree of anisotropy $D A$ of the

178 VOI, where $D A=1-\left(\frac{E_{\min }}{E_{\max }}\right)$. It was also cleverly pointed out in Harrigan and Mann

179 (1984) [35] that the coefficients of Eq. (3) can equally be given as a dimensionless tensor,

180 which they name the materials anisotropy tensor $M$, where $M=\left[\begin{array}{ccc}A & D & E \\ D & B & F \\ E & F & C\end{array}\right]$. Given that

$181 M$ is indiscriminate towards the material to which it is being applied, it is proposed in this

182 study that it could be used as an analog to the tensorial component of Eq. (2). From

183 within the constructs of the commercially available CTan software for micro-CT analysis, 
184 all the variables described above (i.e. $l_{1}, l_{2}, l_{3}, \vec{e}_{1}, \vec{e}_{2}, \vec{e}_{3}, E_{1}, E_{2}, E_{3}, D A$, and $M$ ) can be 185 calculated and given as outputs as part of a 3-D stereological analysis, further 186 demonstrating the advantages of micro-CT.

187 To more plainly demonstrate and test the applicability of the CTan 3-D 188 stereological analysis for application to problems concerning crack orientation and 189 distribution in solids, two test-cases were created and are presented here. The first, a 190 stack of binary images alternating in layers of pixel values set to either 0 or 1 , and

191 oriented such that the 1 layers were perpendicular to the $\mathrm{x}_{3}$ axis is shown in the upper 192 panel of Figure X. The second example shown in the lower panel of Figure X, is the 193 same as that shown in the upper panel, but rotated 90 degrees, such that the layers with 194 pixel values equal to 1 are parallel to the $x_{3}$ axis. These binary matrices were created 195 such that they were 100x100x100 pixels in size, with each pixel equaling $100 \mu \mathrm{m}$ in 196 resolution, resulting in a $1 \mathrm{~cm}$ cube. The stacks were analyzed with the 3-D stereological 197 analysis in CTan, as discussed above.

198 As would be expected, it was found that the eigenvectors with the minimum mean 199 intercept length were normal to the layering of the matrix, while the other two mean 200 intercept lengths were much larger and nearly identical. Also shown in the upper and 201 lower panels of Figure X, are illustrations of the best fitting ellipsoid about the mean 202 intercept lengths, representing the obvious nature of the layering and corresponding 203 anisotropy. Calculated values for the mean intercept length, eigenvectors, and 204 eigenvalues are also given in this figure. 
208 Following Cole (1979) [36], right-cylindrical specimens of pure polycrystalline 209 ice (using Milli-Q 18.2 $\mathrm{M} \Omega \mathrm{cm}^{-1}$ deionized water) were created via a radial freezing 210 technique, where $0.8 \mathrm{~mm}$ seed grains were loaded into an aluminum mold, flooded with 211 chilled and deaerated water, and then slowly frozen radially from the outside-inwards via 212 a temperature controlled glycol wrap placed around the exterior of the mold. Following 213 this approach, polycrystalline specimens of ice with a mean grain diameter of $1 \mathrm{~mm}$ and a 214 cylindrical aspect ratio of 3:1 were created. An example of the initial polycrystalline 215 microstructure is shown in Figure 1, as photographed between crossed polarizing filters. 216 An initial microstructure of randomly oriented grains ranging from $0.8-1.2 \mathrm{~mm}$ in

217 diameter was obtained, as ascertained with polarized light microscopy and a manual 218 calculation of the mean intercept length [37]. Following Cole (1979) [36], this initial 219 microstructure was found to be reproducible. Specimen dimensions were $3.8 \mathrm{~cm}$ in 220 diameter by $11.4 \mathrm{~cm}$ in length.

$222 \quad 3.2$ Mechanical Testing

223 Uniaxial compression tests were carried out using a MTS servo-hydraulic testing 224 apparatus housed in a cold room. At a test temperature of $-10^{\circ} \mathrm{C}\left(+/-0.2^{\circ} \mathrm{C}\right)$, six constant 225 strain rates of $1 \times 10^{-3} \mathrm{~s}^{-1}, 1 \times 10^{-4} \mathrm{~s}^{-1}, 1 \times 10^{-5} \mathrm{~s}^{-1}, 5 \times 10^{-6} \mathrm{~s}^{-1}, 2.5 \times 10^{-6} \mathrm{~s}^{-1}$, and $1 \times 10^{-6} \mathrm{~s}^{-1}$ were 226 applied. At a test temperature of $-20^{\circ} \mathrm{C}\left(+/-0.2^{\circ} \mathrm{C}\right)$, two constant strain rates of $1 \times 10^{-5} \mathrm{~s}^{-1}$ 227 and $1 \times 10^{-6} \mathrm{~s}^{-1}$ were applied. All compression tests were terminated at an engineering 228 strain of $5 \%$ as the peak stress had always been obtained by that point. It should also be 229 noted that, apart from the samples tested at $1 \times 10^{-3} \mathrm{~s}^{-1}$, these are the same samples as those 
230 used for the mechanical testing data shown in Hammonds \& Baker (2016) [38] for pure

231 polycrystalline ice, thus the results from the mechanical tests will not be repeated here.

232 At the completion of each mechanical test, two cross-sectional volumes of ice were cut

233 with a high precision bandsaw for damage analysis via micro-CT that were at least $2 \mathrm{~cm}$

234 in height and $3.8 \mathrm{~cm}$ in diameter. This was done for two specimens per each applied

235 strain rate and temperature, resulting in a total of 32 cross-sectional volumes to be

236 analyzed via micro-CT. Between the periods of mechanical testing and micro-CT

237 analysis, all samples were stored in a $-30^{\circ} \mathrm{C}\left(+/-0.2^{\circ} \mathrm{C}\right)$ cold room to prevent any

238 significant changes in the microstructure.

2403.3 X-ray Computed Micro-Tomography (micro-CT)

241 For the damage analysis and imaging of cracks, a Bruker Skyscan 1172 desktop

242 micro-CT housed in a $-10^{\circ} \mathrm{C}$ cold room was used. For all samples, the $\mathrm{X}$-ray beam was

243 set to $40 \mathrm{kV}$ and $250 \mathrm{~mA}$. The sample was placed on the scanning stage such that the

244 long cylindrical axis ( $\mathrm{z}$-axis or $\mathrm{x}_{3}$ ) was oriented perpendicular to the $\mathrm{X}$-ray beam, as

245 shown in Figure 3. The sample was then rotated $180^{\circ}$ in the $\mathrm{x}-\mathrm{y}$ plane (or $\mathrm{x}_{1}-\mathrm{x}_{2}$ plane) at

$2460.7^{\circ}$ increments while being scanned. Attenuation profiles were captured using a $1.3 \mathrm{Mp}$,

247 12-bit, cooled CCD Hamamatsu camera. A $15 \mu \mathrm{m}$ resolution was used, allowing for the 248 actual scanned inner volume of the sample to be $1.9 \mathrm{~cm}$ in diameter by $1.5 \mathrm{~cm}$ tall. If

249 using software to stitch together reconstructions, it would be possible to scan the entire

250 sample, but that was not done here, as the volume of interest (VOI) from one individual

251 scan was large enough to capture what is thought to be a representative volume of cracks 252 and orientations. 
254 performed using NRecon software, which included thermal drift correction, post

255 alignment, removal of ring artifacts, Gaussian pixel smoothing, and beam hardening

256 correction. Because the resulting images from this reconstruction are on a 0-255

257 grayscale, they must then be converted to binary images, such that they can be analyzed

258 as individual pixels made up of either 0's (ice) or 1's (air/crack) on a binary scale. To

259 allow for absolute control of the binary conversion, grayscale images were converted

260 using Matlab's image processing toolbox, although CTan could have also been used. As

261 per visual inspection, this yielded satisfactory results overall, but it should be noted that

262 the threshold chosen for the conversion will ultimately create noise in some images while

263 potentially deleting parts of the objects of interest in others. Therefore, for each sample

264 scanned, a representative set of images must be visually inspected upon conversion to

265 assure that the objects of interest (cracks) are being accounted for while the noise is kept

266 to a minimum. To illustrate this point, a typical binary conversion is shown in Figure 2,

267 where two different thresholds were chosen for the same 2-D grayscale image taken from

268 a sample tested at $-10^{\circ} \mathrm{C}$ and $1 \times 10^{-3} \mathrm{~s}^{-1}$ (Figure 2a). Notice that neither images Figure $2 \mathrm{~b}$

269 or Figure 2c perfectly capture the full and unbiased extent of the micro-cracks observed

270 in the grayscale image, but Figure $2 \mathrm{~b}$ would certainly yield more accurate results than

271 Figure 2c when performing a 2-D or 3-D quantitative analysis based on the number and

272 location of binary pixels ( 0 or 1$)$, thus a threshold is manually chosen that is applied to

273 the entire stack of images.

274

275 4. RESULTS \& DISCUSSION 
The most easily obtainable metric for imparted damage $D$ from a binary micro-CT

278 reconstruction is a simple cavity volume fraction of air to ice (i.e. $D=V_{\text {air }} / V_{\text {ice }}$ ) from

279 within a given VOI. This approach can be useful if considering the evolution of damage

280 within a material over periods of varying stress, strain, or time [39,8]. Expressed as a

281 percentage, $D$ is shown in Figure 4 as the mean value for all scans performed of all

282 samples over a given strain rate. In this figure, $D$ monotonically increases upon

283 increasing the applied strain rate at $-10^{\circ} \mathrm{C}$ and $-20^{\circ} \mathrm{C}$, as would be expected. What is

284 missing from this analysis, however, is additional information pertaining to the number of

285 cracks, their orientation, their distribution about the volume, and whether or not any

286 anisotropy is present, as may also be expected from a compression test conducted in a

287 uniaxial mode [34]. Although an individual object analysis can be performed within

288 CTan, such that each individual crack becomes an object, the focus here is on only the

289 distribution of cracks about the VOI and the presence of any anisotropy within this

290 distribution of crack orientations. To further elucidate this point, Figure 5 shows images

291 for tests conducted at $1 \times 10^{-4} \mathrm{~s}^{-1}$ and $1 \times 10^{-5} \mathrm{~s}^{-1}$ at temperatures of $-10^{\circ} \mathrm{C}$ and $-20^{\circ} \mathrm{C}$,

292 respectively, when viewed along the loading direction $\mathrm{x}_{3}$ (top view) vs. perpendicular to

293 the loading direction $\mathrm{x}_{1}$ or $\mathrm{x}_{2}$ (side view). In these images, a clear example of anisotropy

294 exists in the crack orientation, such that most cracks formed parallel to the loading

295 direction. This trend was apparent throughout all specimens analyzed with the micro-CT,

296 except at the lower strain rates $\left(\leq 5 \times 10^{-6} \mathrm{~s}^{-1}\right)$, where virtually no cracking was observed.

297 Previously, such information about the 3-D anisotropy of damage within a specimen

298 would have been difficult to obtain, normally extrapolated from a 2-D analysis, but with 
micro-CT imagery and reconstruction such characteristics become readily apparent. To

300 account for this anisotropy in calculations, the implementation of a crack density tensor,

301 and not just a scalar variable, would be most beneficial. Additional micro-CT images and

302 views, as well as micro-CT animations, are provided in the supplementary material.

303

304 4.2 Extension of the Crack Density Tensor

305 In order to account for all $\mathrm{x}, \mathrm{y}$, and $\mathrm{z}$ components of the crack orientation

306 distribution and resultant anisotropy, a variant of $\alpha_{3-D}$ (Eq. 2) is proposed here, which to

307 the author's knowledge has never before been proposed or applied to ice. Key to this

308 variant of $\alpha_{3-D}$, is the 3-D stereological analysis introduced in Section 2.2 and the

309 concept of a mean intercept length. It could also be interpreted as an inverse crack

310 density tensor, but it will be shown in this section that the materials anisotropy tensor is

311 of an equal measure. Given that all crack lengths and orientations within a particular

312 VOI can be reasonably and statistically represented as an ellipsoid, it would seem that Eq.

313 (2) could be modified such that $a_{i}$ becomes $l_{i}$ and $\vec{n} \vec{n}$ becomes $\vec{e}_{i} \vec{e}_{i}$, where $l_{i}$ is the mean

314 intercept length in each of the three orthogonal directions of the ellipsoid, represented by

315 the three eigenvectors $\vec{e}_{i}$. Making this substitution yields a volume-averaged and inverse

316 variant of the crack density tensor $\left\langle\alpha_{3-D}\right\rangle_{V}$, where Eq. (2) becomes that given in Eq. (4).

$$
\left\langle\alpha_{3-D}\right\rangle_{V}=\frac{1}{V}\left(l_{1}^{3} \vec{e}_{1} \vec{e}_{1}+l_{2}^{3} \vec{e}_{2} \vec{e}_{2}+l_{3}^{3} \vec{e}_{3} \vec{e}_{3}\right)
$$

320 In this equation, the importance of crack orientation and length is conserved, but it should 321 be noted that $\vec{e}_{i}$ is in the same orientation as that in which the mean linear intercept length 
$322 l_{i}$ was measured. Again, if a scalar representation of crack density is desired, then $323 \operatorname{tr}\left\langle\alpha_{3-D}\right\rangle_{V}$ yields such a result, as the first invariant of $\left\langle\alpha_{3-D}\right\rangle_{V}$. To show the

324 effectiveness of $\left\langle\alpha_{3-D}\right\rangle_{V}$ in demonstrating the anisotropy in damage observed over all 325 compression tests, the ratios $\left\langle\alpha_{3-D}\right\rangle_{V_{22}} /\left\langle\alpha_{3-D}\right\rangle_{V_{11}},\left\langle\alpha_{3-D}\right\rangle_{V_{33}} /\left\langle\alpha_{3-D}\right\rangle_{V_{11}}$, and

$326\left\langle\alpha_{3-D}\right\rangle_{V_{33}}\left\langle\left\langle\alpha_{3-D}\right\rangle_{V_{22}}\right.$ are shown in Figure $6 \mathrm{a}$ as functions of the applied strain rate at -

$32710^{\circ} \mathrm{C}$. For comparison to the material anisotropy tensor $M$ (see Section 2.2), the ratios

$328 M_{22} / M_{11}, M_{33} / M_{11}$, and $M_{33} / M_{22}$ are also shown in Figure $6 \mathrm{~b}$. In these figures, it can

329 be seen that very little anisotropy exists between $l_{1}$ and $l_{2}$ and their respective

330 orientations $\vec{e}_{1}$ and $\vec{e}_{2}$. However, between $l_{1}$ and $l_{3}$ and $l_{2}$ and $l_{3}$, and their respective

331 orientations an anisotropy exists, as for a perfectly isotropic medium all ratios would

332 have been equal to 1. Also, and perhaps not surprisingly, the trends observed in Figures

$3336 \mathrm{a}$ and $6 \mathrm{~b}$ are quite similar, as they are both based on the mean intercept length and

334 orientation, suggesting that $M$ is a direct measurement of anisotropy in crack orientation,

335 without the need of calculating $\left\langle\alpha_{3-D}\right\rangle_{V}$. If allowing for this substitution, Eq. (4) then

336 gives

337

$$
\left\langle\alpha_{3-D}\right\rangle_{M}=\frac{1}{V}\left(l_{1}^{3} M+l_{2}^{3} M+l_{3}^{3} M\right)
$$

339 which simplifies to

340

$$
\left\langle\alpha_{3-D}\right\rangle_{M}=\frac{M}{V}\left(l_{1}^{3}+l_{2}^{3}+l_{3}^{3}\right)
$$


343 where $\left\langle\alpha_{3-D}\right\rangle_{M}$ remains a unitless second-rank tensor representation of the crack

344 orientation and mean intercept length. For comparison in tensor form, one example of

$345\left\langle\alpha_{3-D}\right\rangle_{V}$ and $\left\langle\alpha_{3-D}\right\rangle_{M}$ from a single compression test at each strain rate and at $-10^{\circ} \mathrm{C}$ are

346 given in Table 1.

3484.3 Crack Density Distribution

349 While both the traditional crack density tensor and the proposed variant of the

350 crack density tensor give information about the anisotropy of crack orientation, they

351 equally lack information about the three-dimensional distribution of cracks about the

352 entire VOI. For this, a simple eigenanalysis is more useful. In addition to obtaining

353 eigenvectors with CTan, eigenvalues $\left(E_{1}, E_{2}, E_{3}\right)$ are also computed. These eigenvalues

354 correlate directly to the coefficients of the ellipsoid given in Eq. (3). Because the

355 eigenvalues represent the geometric transformation of the axes of the ellipsoid $\left(\vec{e}_{1}, \vec{e}_{2}, \vec{e}_{3}\right)$

356 back to the principal axes of the scanned volume $\left(x_{1}, x_{2}, x_{3}\right)$ of unit length, a larger

357 eigenvalue corresponds to a greater degree of "stretching" or "compressing" of that axis.

358 Thus, large eigenvalues correspond to large geometric transformations of that axis.

359 Figure 7 a shows the mean eigenvalues $\left(\overline{E_{1}}, \overline{E_{2}}, \overline{E_{3}}\right)$ calculated for each constant strain rate

360 test at $-10^{\circ} \mathrm{C}$. In this figure, the similarities in $\overline{E_{1}}$ and $\overline{E_{2}}$ demonstrate the relative isotropy

361 observed with respect to the $\mathrm{x}_{1}$ and $\mathrm{x}_{2}$ axes. At the same time, decreasing values of $\overline{E_{3}}$

362 with increasing strain rate demonstrates an increasing number of intercepts in $x_{3}$. This

363 occurs because at low strain rates, cracking begins in only select regions and is not

364 uniform over the entire sample volume. This creates an anisotropy in the crack

365 orientation and relative distribution about the VOI, as evidenced by taking the trace of 
366 Eq. (5), $\operatorname{tr}\left\langle\alpha_{3-D}\right\rangle_{M}$, as shown in Figure 7b. Here, the decreasing trend of $\operatorname{tr}\left\langle\alpha_{3-D}\right\rangle_{M}$

367 with increasing strain rate provides evidence of the crack distribution becoming more 368 uniform as more cracks begin filling out the VOI.

3704.4 Discussion

371 The results presented in this section are thought to be of significance, as similar 372 previous laboratory studies investigating the mechanical properties of polycrystalline ice

$373[8,14,16]$ have not yet been able to quantify many of the three-dimensional characteristics

374 of the damage which they had observed. For example, quantifying the crack shape,

375 connectivity, orientation, and spatial distribution of cracks could allow for a more

376 rigorous 3-D model to be developed relating the elasticity and porosity of ice $[33,8]$.

377 Although this was beyond the scope of this study, it has now been shown to be well

378 within the realm of what is possible with micro-CT. Additionally, as shown in Figure 4

379 for the data collected at $-10^{\circ} \mathrm{C}$, it is possible that an exponential relationship may exist

380 between the rate of cavity growth and the imposed strain rate in polycrystalline ice. If

381 these experiments were to be repeated as a function of strain instead of strain rate, for

382 instance, it is possible with the approach presented here that such information could lend

383 additional insight into the mechanics of the cavity development and whether there is a

384 strain dependency indicative of a more ductile or brittle regime of deformation occurring

385 locally [40]. With the introduction of the quantitative techniques presented in this study,

386 combined with the many other recent advances in the quantification of damage and

387 deformation in other polycrystalline materials via micro-CT [41], it is thought that a new

388 paradigm in the laboratory study of polycrystalline ice is possible. 


\section{CONCLUSIONS}

391 It has been shown that the use of X-ray computed micro-tomography can be

392 extended to the study of damage and the onset of micro-cracking in polycrystalline ice.

393 This was demonstrated through the detailed analysis of a set of experiments where

394 laboratory-prepared samples of polycrystalline ice, of a $1 \mathrm{~mm}$ mean grain diameter, were

395 strained at six strain rates spanning four orders of magnitude and two different test

396 temperatures. It was found that a clear anisotropy exists in both the orientation and

397 distribution of micro-cracks and that these features could be quantified and measured via

398 existing and commercially available software for micro-CT. Additionally, it was found

399 that two individual measurements of anisotropy are necessary for measuring the

400 anisotropy of crack orientation vs. crack distribution, and that these can both be

401 determined with a mean intercept length analysis and quantified with the materials

402 anisotropy tensor, as first given in Harrigan \& Mann (1984) [35]. Using this approach, a

403 new and alternative form of the traditional crack density tensor was introduced. In future

404 work, the mechanisms responsible for the damage observed in these test specimens, as

405 well as their connectivity as a function of strain, will be addressed.

406

407

408

409

410

411 


\section{ACKNOWLEDGEMENTS}

413 This work was supported by NSF grant \# 1141411. The authors thank Professor Carl

414 Renshaw for his helpful comments and acknowledge the use of the Dartmouth Ice

415 Research Laboratory for this work (Director E.M. Schulson).

416 


\section{FIGURES}

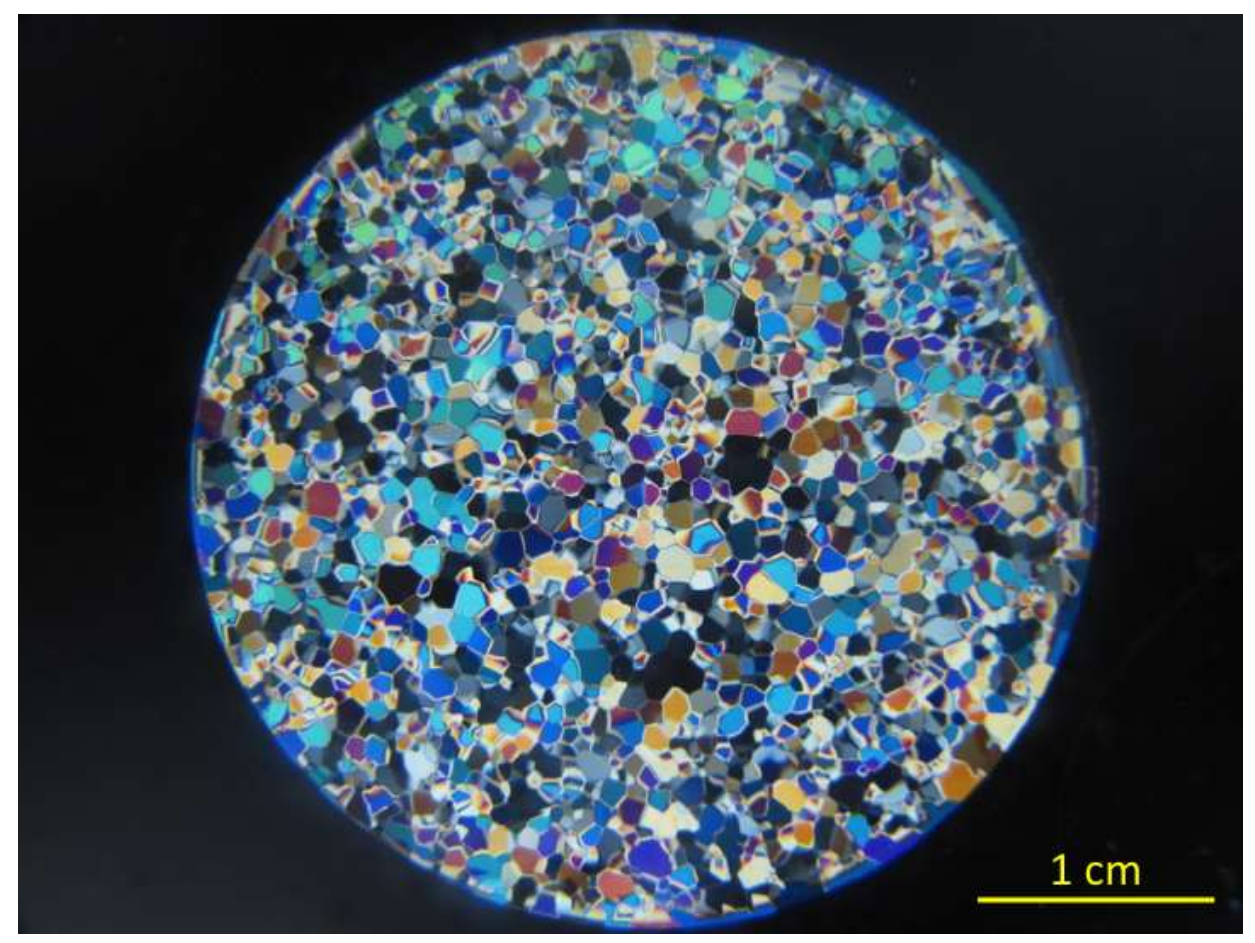

Figure 1. Initial microstructure of a cylindrical specimen prepared for mechanical testing as viewed through crossed polarizing filters. Mean grain diameters were typically on the order of $1 \mathrm{~mm}$. Adapted from Hammonds \& Baker 2016. 

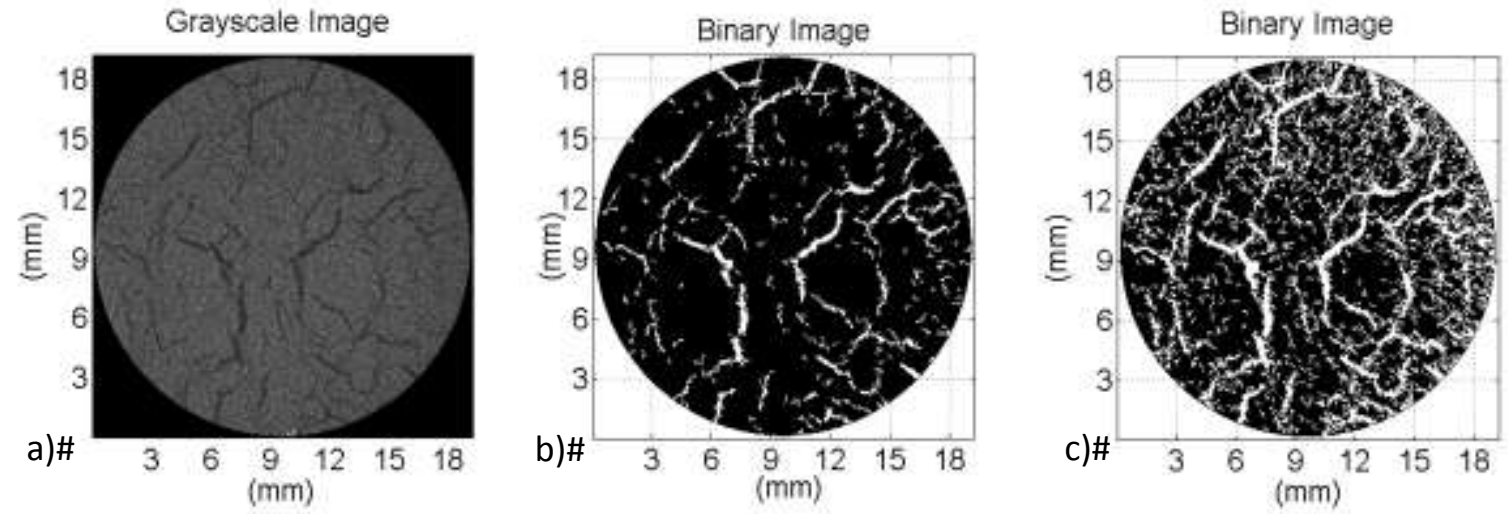

Figure 2. Conversion of grayscale image generated by micro-CT reconstruction (a) to an optimal binary image (b) and an example of a much less optimal binary image (c). Specimen was strained to $5 \%$ at a constant strain rate of $1 \times 10^{-3} \mathrm{~s}^{-1}$ at $-10^{\circ} \mathrm{C}$. View is looking down the loading axis $\left(\mathrm{x}_{3}\right)$. 

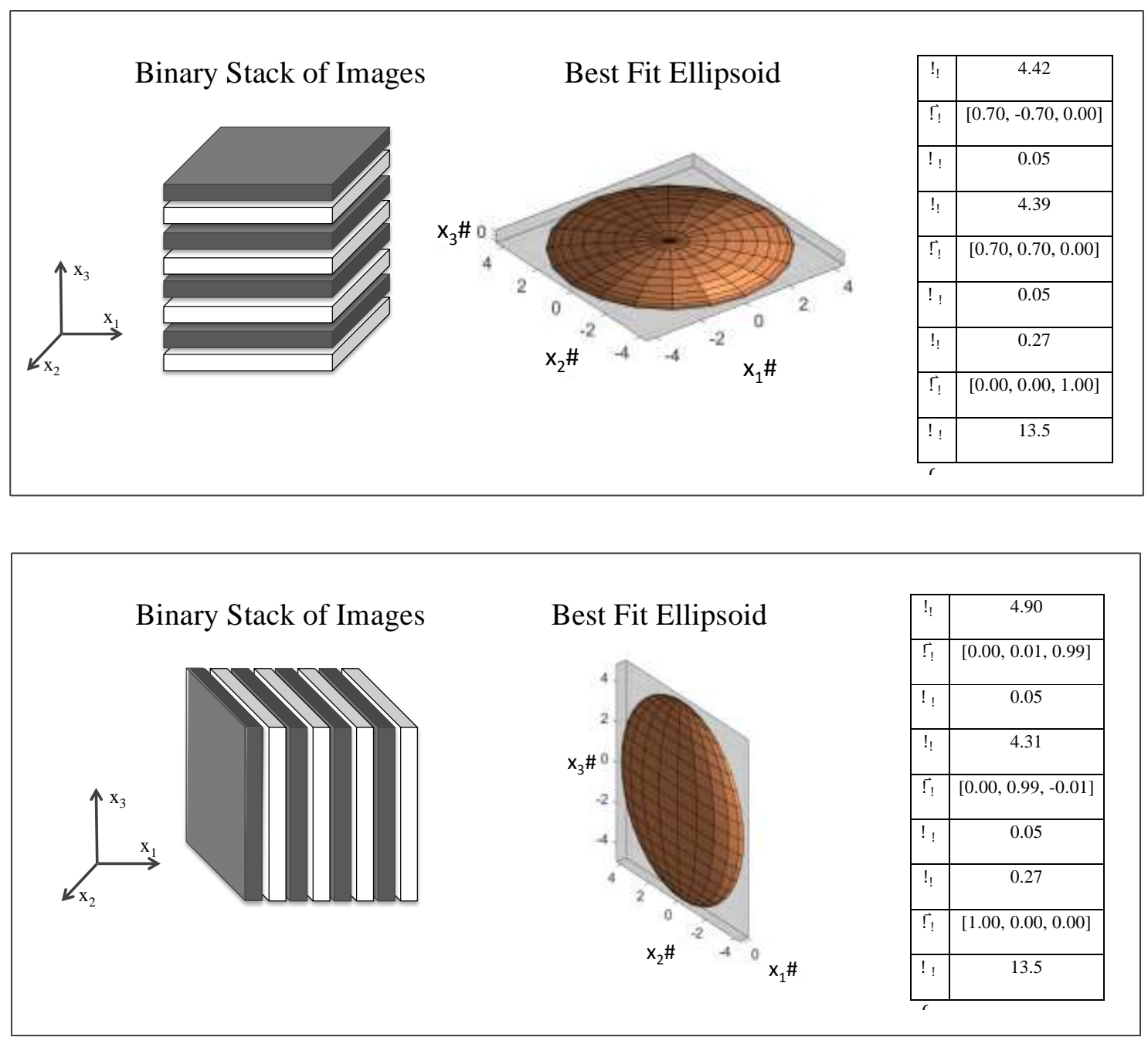

Figure 3. 


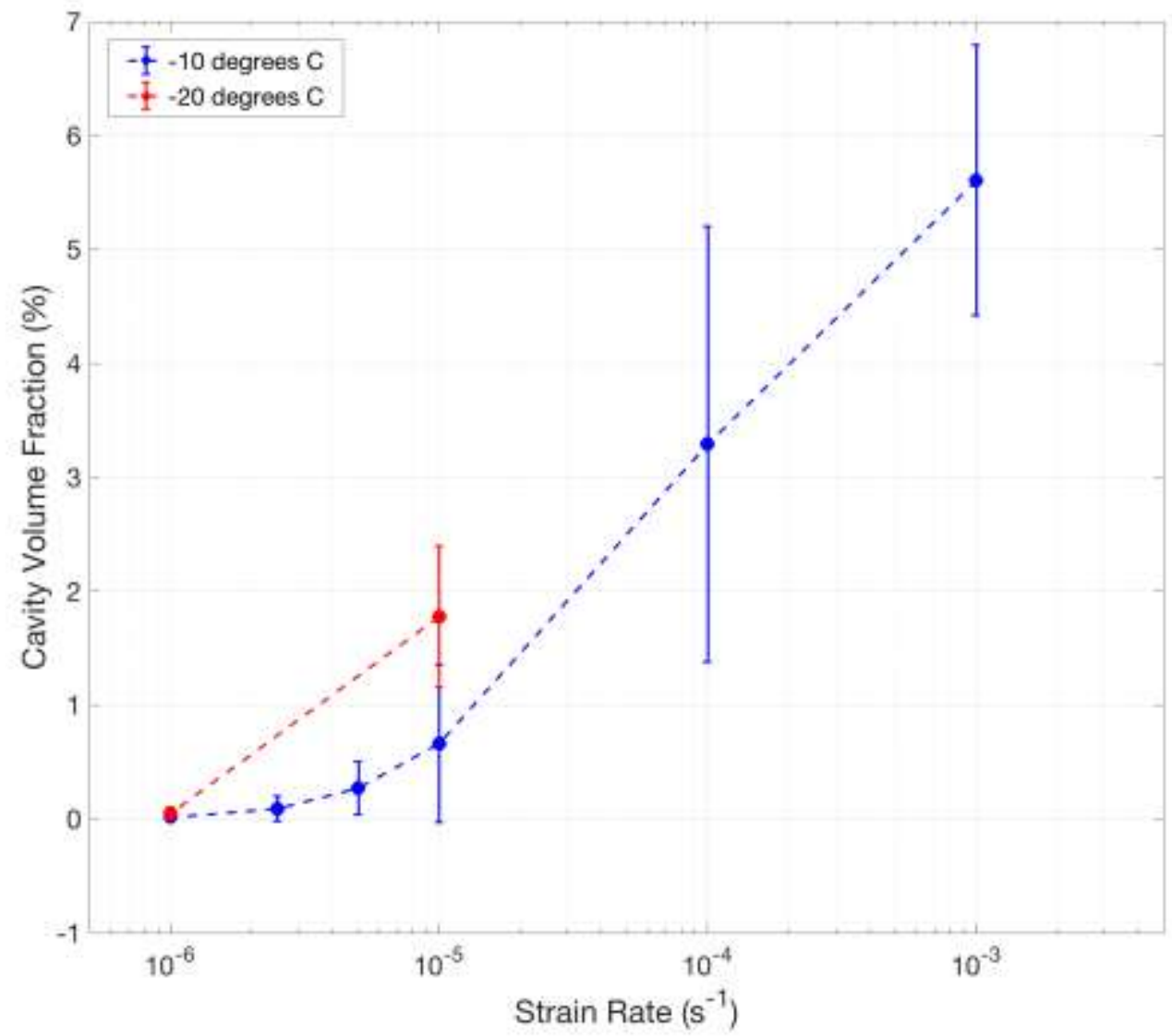

Figure 4. Mean volume fraction of air to ice, calculated from 3-D binary reconstructions over all strain rates and temperatures. The mean cavity volume fraction could be considered as a metric for the volumetric crack density. Error bars represent one standard deviation from the mean with $n=4$ samples. 

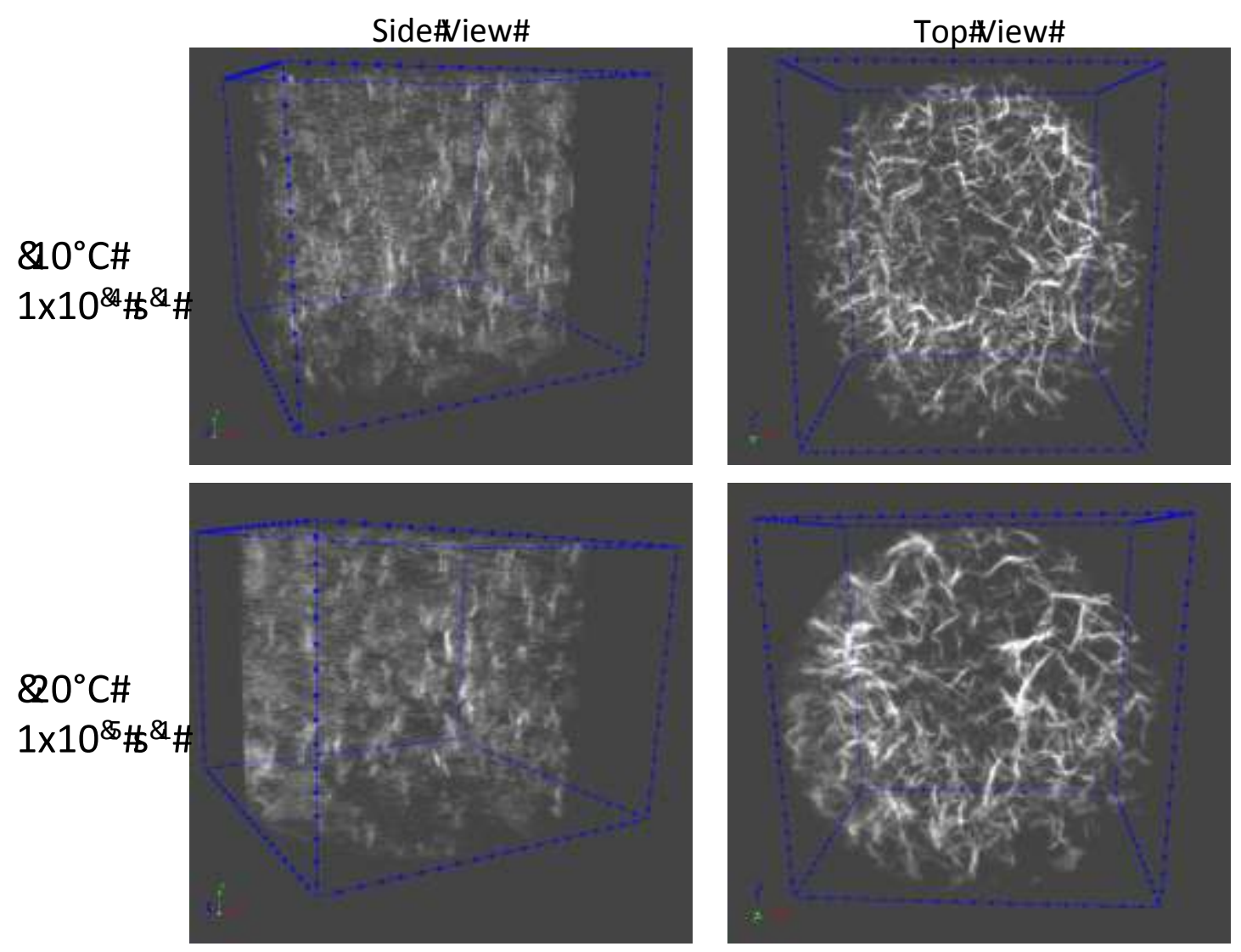

Figure 5. Micro-CT reconstructions of binary images taken from specimens tested at constant strain rates of $1 \times 10^{-4} \mathrm{~s}^{-1}$ at $-10^{\circ} \mathrm{C}$ (upper) and $1 \times 10^{-3} \mathrm{~s}^{-1}$ at $-20^{\circ} \mathrm{C}$ (lower). This view was generated with micro-CT software that maximally limits attenuation, giving a "see-through" appearance. Distance between blue dots encompassing image denote 1 mm scale. Top view is looking down the loading direction $\left(\mathrm{x}_{3}\right)$. 

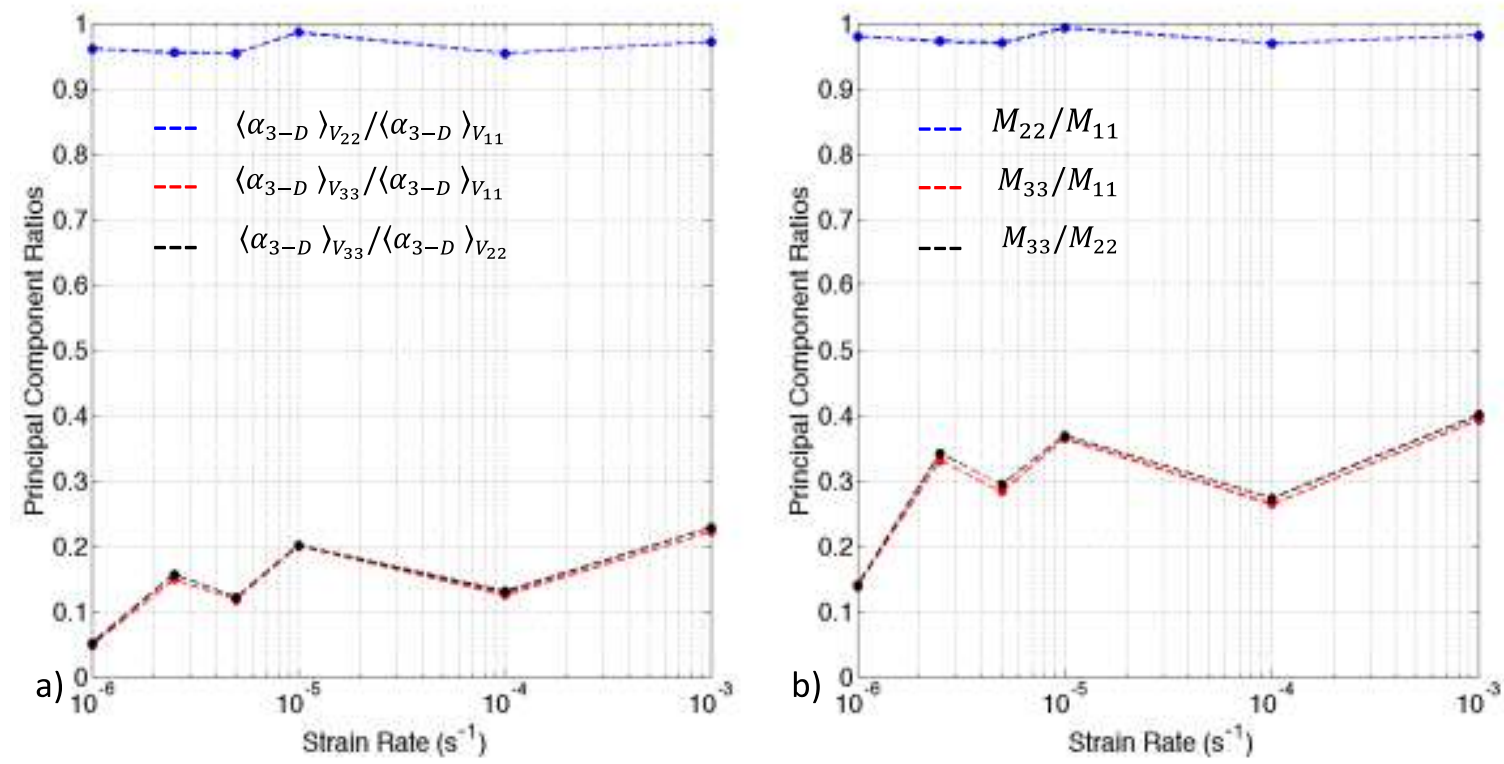

Figure 6. Principal component ratios from (a) the proposed volumetric crack density tensor $\left\langle\alpha_{3-D}\right\rangle_{V}$ (see Eq. 4) and (b) the materials anisotropy tensor $M$ (see Section 2.2) at $-10^{\circ} \mathrm{C}$.
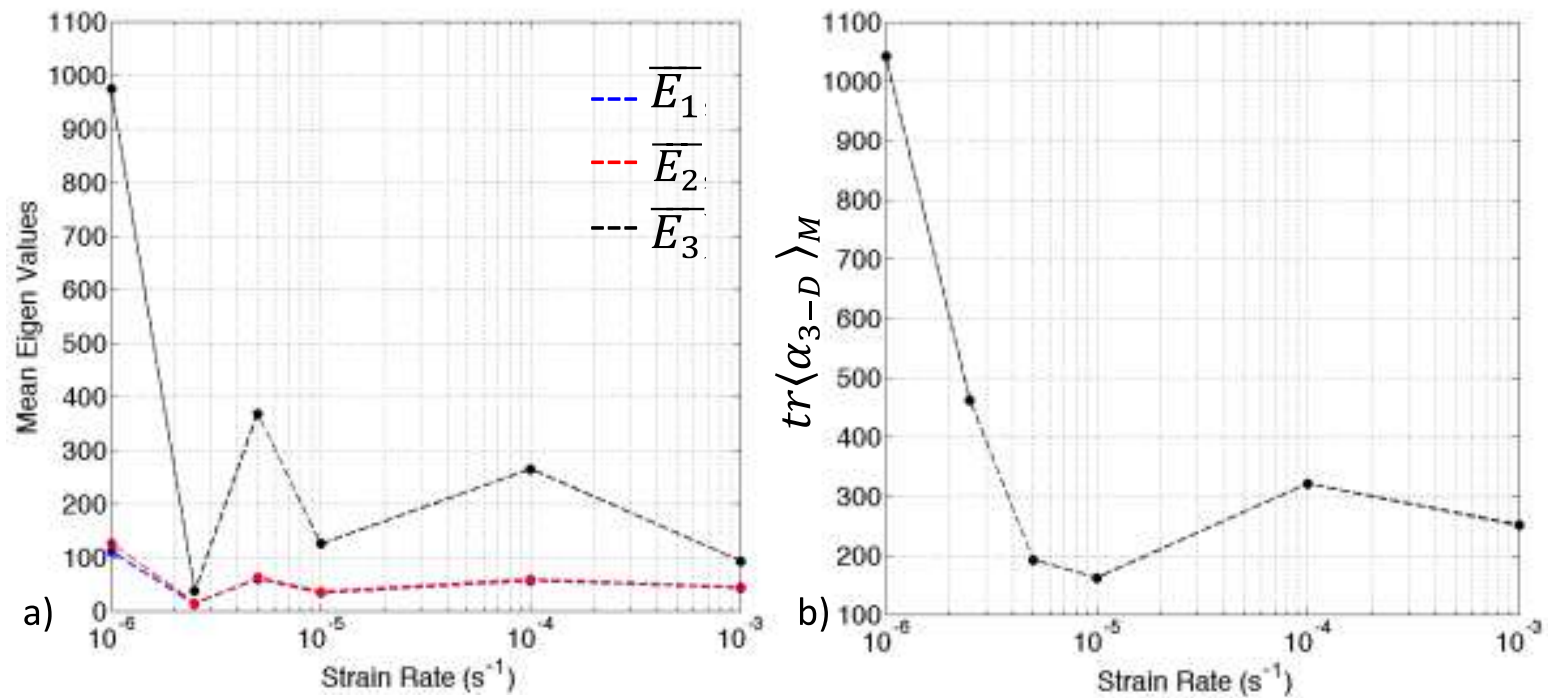

Figure 7. Mean eigenvalues for all constant strain rate tests at $-10^{\circ} \mathrm{C}$ (a) and $\operatorname{tr}\left\langle\alpha_{3-D}\right\rangle_{M}$ for all constant strain rate tests (b). 


\begin{tabular}{|c|c|c|}
\hline Strain Rate & $\left\langle\alpha_{3-D}\right\rangle_{V}=\frac{1}{V}\left(l_{1}^{3} \vec{e}_{1} \vec{e}_{1}+l_{2}^{3} \vec{e}_{2} \vec{e}_{2}+l_{3}^{3} \vec{e}_{3} \vec{e}_{3}\right)$ & $\left\langle\alpha_{3-D}\right\rangle_{M}=\frac{M}{V}\left(l_{1}^{3}+l_{2}^{3}+l_{3}^{3}\right)$ \\
\hline $1 \times 10^{-3} \mathrm{~s}^{-1}$ & $\begin{array}{rrr}8.30 \mathrm{e}-07 & 1.79 \mathrm{e}-08 & -3.79 \mathrm{e}-09 \\
1.79 \mathrm{e}-08 & 8.09 \mathrm{e}-07 & 2.33 \mathrm{e}-09 \\
-3.79 \mathrm{e}-09 & 2.33 \mathrm{e}-09 & 2.64 \mathrm{e}-07\end{array}$ & $\begin{array}{rrr}8.26 \mathrm{e}-05 & -2.44 \mathrm{e}-06 & -8.21 \mathrm{e}-07 \\
-2.44 \mathrm{e}-06 & 8.40 \mathrm{e}-05 & 1.28 \mathrm{e}-06 \\
-8.21 \mathrm{e}-07 & 1.28 \mathrm{e}-06 & 1.77 \mathrm{e}-04\end{array}$ \\
\hline $1 \times 10^{-4} s^{-1}$ & $\begin{array}{rrr}1.23 \mathrm{e}-06 & -2.74 \mathrm{e}-08 & 3.61 \mathrm{e}-09 \\
-2.74 \mathrm{e}-08 & 1.16 \mathrm{e}-06 & 2.20 \mathrm{e}-09 \\
3.61 \mathrm{e}-09 & 2.20 \mathrm{e}-09 & 2.28 \mathrm{e}-07\end{array}$ & $\begin{array}{rrr}8.76 \mathrm{e}-05 & 2.72 \mathrm{e}-06 & -8.68 \mathrm{e}-07 \\
2.72 \mathrm{e}-06 & 9.10 \mathrm{e}-05 & -1.32 \mathrm{e}-06 \\
-8.68 \mathrm{e}-07 & -1.32 \mathrm{e}-06 & 2.69 \mathrm{e}-04\end{array}$ \\
\hline $1 \times 10^{-5} s^{-1}$ & $\begin{array}{rrr}4.38 \mathrm{e}-07 & -1.11 \mathrm{e}-08 & -5.34 \mathrm{e}-10 \\
-1.11 \mathrm{e}-08 & 4.62 \mathrm{e}-07 & 2.67 \mathrm{e}-10 \\
-5.34 \mathrm{e}-10 & 2.67 \mathrm{e}-10 & 7.32 \mathrm{e}-08\end{array}$ & $\begin{array}{rrr}6.47 \mathrm{e}-05 & 2.09 \mathrm{e}-06 & -1.96 \mathrm{e}-07 \\
2.09 \mathrm{e}-06 & 6.24 \mathrm{e}-05 & 4.31 \mathrm{e}-07 \\
-1.96 \mathrm{e}-07 & 4.31 \mathrm{e}-07 & 2.13 \mathrm{e}-04\end{array}$ \\
\hline $5 \times 10^{-6} \mathrm{~s}^{-1}$ & $\begin{array}{rrr}4.85 \mathrm{e}-06 & -3.18 \mathrm{e}-07 & 7.58 \mathrm{e}-09 \\
-3.18 \mathrm{e}-07 & 4.64 \mathrm{e}-06 & 1.29 \mathrm{e}-08 \\
7.58 \mathrm{e}-09 & 1.29 \mathrm{e}-08 & 1.01 \mathrm{e}-06\end{array}$ & $\begin{array}{rrr}1.41 e-04 & 1.28 \mathrm{e}-05 & -1.88 \mathrm{e}-06 \\
1.28 \mathrm{e}-05 & 1.45 \mathrm{e}-04 & -1.13 \mathrm{e}-06 \\
-1.88 \mathrm{e}-06 & -1.13 \mathrm{e}-06 & 3.98 \mathrm{e}-04\end{array}$ \\
\hline $2.5 \times 10^{-6} \mathrm{~s}^{-1}$ & $\begin{array}{rrr}1.76 \mathrm{e}-07 & -4.15 \mathrm{e}-08 & 2.73 \mathrm{e}-10 \\
-4.15 \mathrm{e}-08 & 1.73 \mathrm{e}-07 & 6.06 \mathrm{e}-11 \\
2.73 \mathrm{e}-10 & 6.06 \mathrm{e}-11 & 2.16 \mathrm{e}-08\end{array}$ & $\begin{array}{rrr}4.68 \mathrm{e}-05 & 1.50 \mathrm{e}-05 & -2.27 \mathrm{e}-07 \\
1.50 \mathrm{e}-05 & 4.72 \mathrm{e}-05 & -5.37 \mathrm{e}-07 \\
-2.27 \mathrm{e}-07 & -5.37 \mathrm{e}-07 & 1.83 \mathrm{e}-04\end{array}$ \\
\hline $1 \times 10^{-6} \mathrm{~s}^{-1}$ & $\begin{array}{lll}1.72 \mathrm{e}-07 & 9.85 \mathrm{e}-09 & 8.58 \mathrm{e}-10 \\
9.85 \mathrm{e}-09 & 1.90 \mathrm{e}-07 & 5.10 \mathrm{e}-10 \\
8.58 \mathrm{e}-10 & 5.10 \mathrm{e}-10 & 1.20 \mathrm{e}-08\end{array}$ & $\begin{array}{rrr}4.64 \mathrm{e}-05 & -3.25 \mathrm{e}-06 & -1.20 \mathrm{e}-06 \\
-3.25 \mathrm{e}-06 & 4.35 \mathrm{e}-05 & -2.36 \mathrm{e}-06 \\
-1.20 \mathrm{e}-06 & -2.36 \mathrm{e}-06 & 2.74 \mathrm{e}-04\end{array}$ \\
\hline
\end{tabular}

Table 1. Variants of the crack density tensor calculated with Eq. (4) and Eq. (5) from one test at each constant applied strain rate at $-10^{\circ} \mathrm{C}$. 